\title{
Kaisa Häkkinen : Spreading the Written Word
}

SKS, 2015

\section{Lea Lagerblom}

Traducteur : Aleksi Moine

\section{OpenEdition}

\section{Journals}

Édition électronique

URL : https://journals.openedition.org/efo/7028

DOI : $10.4000 /$ efo. 7028

ISSN : 2275-1947

\section{Éditeur}

INALCO

Édition imprimée

ISBN : 978-2-343-12463-6

ISSN : 0071-2051

\section{Référence électronique}

Lea Lagerblom, « Kaisa Häkkinen : Spreading the Written Word », Études finno-ougriennes [En ligne], 48 2017, mis en ligne le 17 juillet 2017, consulté le 08 juillet 2021. URL : http://journals.openedition.org/ efo/7028; DOI : https://doi.org/10.4000/efo.7028

Ce document a été généré automatiquement le 8 juillet 2021

\section{(c) (†) 8}

Études finno-ougriennes est mis à disposition selon les termes de la Licence Creative Commons Attribution - Pas d'Utilisation Commerciale 4.0 International. 


\section{Kaisa Häkkinen : Spreading the Written Word}

SKS, 2015

Lea Lagerblom

Traduction : Aleksi Moine

1 Mikael Agricola (vers 1510-1557), de son vrai nom Mikael Olavinpoika, fils d'un paysan de Pernaja, est devenu, grâce à son talent, son savoir et ses relations, la figure de proue de la Réforme en Finlande, le directeur de la célèbre école de la cathédrale de Turku, et enfin l'évêque de Turku. Ses neuf ouvrages, parmi lesquels la traduction finnoise du Nouveau Testament, sont les premiers livres imprimés en finnois, et constituent le fondement de la langue finnoise écrite. Mikael Agricola a composé ses ouvrages pour que le peuple puisse, conformément aux principes de la Réforme, entendre et lire la parole de Dieu dans sa propre langue.

2 Spreading the Written Word (SKS, 2015), de Kaisa Häkkinen, professeur émérite de langue finnoise à l'université de Turku, spécialiste de Mikael Agricola, est la première présentation générale de Mikael Agricola et de son œuvre destinée à un public international. L'ouvrage contextualise précisément son sujet, de sorte que l'importance de l'œuvre de Mikael Agricola et les particularités de sa langue sont compréhensibles à un large lectorat. Sa vaste bibliographie en fait également un manuel utile pour les chercheurs, puisqu'il présente de façon détaillée les recherches effectuées sur Mikael Agricola jusqu'à aujourd'hui, ainsi que les ressources disponibles. Le livre est divisé en six chapitres, dont le premier présente le contexte religieux et historicoculturel de l'activité de Mikael Agricola. Dans le deuxième, l'auteure étudie les étapes de sa vie dans l'ordre chronologique et, dans les chapitres 3 et 4 , ses œuvres et leur langue. Le cinquième chapitre de l'ouvrage approfondit les éléments biographiques du deuxième chapitre en présentant notamment les personnes qui ont influencé la vie de Mikael Agricola. Dans le sixième chapitre, l'auteure évalue l'importance de son œuvre et présente l'histoire de la recherche sur Mikael Agricola et la situation contemporaine.

3 Le premier chapitre de l'ouvrage commence par aborder l'époque précédant la Réforme qui a eu lieu au Xvi ${ }^{e}$ siècle, contemporaine de Mikael Agricola et de la mise à l'écrit du 
finnois : l'auteure y décrit le changement de la culture spirituelle à l'époque viking, alors que le christianisme commençait à se diffuser vers le Nord, ainsi que l'arrivée de la culture écrite dans la Finlande médiévale. Le récit historique est rendu vivant par la présence de détails intéressants. Kaisa Häkkinen parle ainsi de la première bibliothèque connue en Finlande, qui appartenait à l'évêque de Finlande Tuomas, et comprenait 58 livres. Elle parle également des lettres sur écorce de bouleau trouvées lors de fouilles archéologiques, qui témoignent de l'activité de la culture écrite au Moyen Âge. Kaisa Häkkinen souligne le rôle central des livres dans l'activité de l'Église dès avant la Réforme, ainsi que l'importance des monastères comme centres de l'activité littéraire. Dans la Finlande médiévale, on importait des livres de l'étranger et on les écrivait et copiait à la main, d'abord sur parchemin, puis également sur papier à partir du XIII ${ }^{\mathrm{e}}$ siècle. Ce n'est qu'à partir de la moitié $\mathrm{du} \mathrm{XV}^{\mathrm{e}}$ siècle que sont apparus les premiers ouvrages imprimés, et le premier livre imprimé pour la Finlande est un missel imprimé à Lübeck en 1488, Missale Aboense.

4 Les langues de l'écriture dans la Finlande médiévale étaient le latin ou le suédois, et le finnois n'était utilisé que pour les noms de personnes et de lieux. D'après Kaisa Häkkinen, toutefois, il est probable que le finnois ait été utilisé dans une certaine mesure pour les besoins de l'Église dès cette époque, mais aucun témoignage n'en a été conservé. Les premiers manuscrits en finnois arrivés jusqu'à nous, comme le Kangasla Missal et la compilation de manuscrits connue sous le nom de Westhin koodeksi, n'ont été rédigés qu'à l'époque de Mikael Agricola ou un peu avant. Kaisa Häkkinen guide le lecteur auprès des manuscrits conservés jusqu'à nos jours: on peut les consulter à la Bibliothèque nationale de Finlande à Helsinki et quelques-uns d'entre eux sont consultables sur Internet, où ils sont disponibles en version électronique. L'ouvrage mentionne également les compilations dans lesquelles les manuscrits ont été publiés.

5 Kaisa Häkkinen esquisse la situation non seulement de la langue écrite mais aussi de la langue parlée dans la Finlande médiévale. Selon elle, on ne peut parler de langue finnoise médiévale dans la mesure où il n'existait pas en Finlande une langue unique et commune, mais de nombreux dialectes. D'un autre côté, Kaisa Häkkinen émet l'hypothèse que, à la suite des contacts linguistiques et à l'assimilation de différentes langues, une nouvelle langue commune générale aurait pu naître dans les villes, en particulier pour les besoins de la religion et de l'Église. D'autres langues étaient également parlées en Finlande : dans le Häme, et légèrement plus au nord, il y avait des communautés de langue same, et au tournant du XIII ${ }^{e}$ siècle les côtes de l'ouest et du sud-ouest de la Finlande ont accueilli des populations suédophones. La bourgeoisie de Turku et de Vyborg par ailleurs comprenait en particulier au XIV siècle un grand nombre de marchands hanséatiques parlant le bas-allemand.

$6 \quad$ La Réforme protestante a eu une influence non négligeable sur les débuts du finnois écrit. Kaisa Häkkinen décrit dans le premier chapitre de son ouvrage le contexte de la Réforme, ses figures prépondérantes, le rôle central de Wittenberg, ainsi que la diffusion de la Réforme au Danemark, en Suède et en Finlande. Elle rappelle que, même si la Réforme a conduit, dans tous ces pays, à un développement important d'une langue écrite, les conditions y étaient plus propices au Danemark et en Suède qu'en Finlande. En effet, le danois et le suédois étaient déjà des langues écrites, contrairement au finnois que l'on commençait tout juste à développer à l'écrit. L'ouvrage décrit la Réforme en Finlande de façon détaillée et esquisse l'état de la littérature de langue 
finnoise, rédigée à la main ou publiée dans les ouvrages de Mikael Agricola. Outre les textes religieux, on écrivait des textes de lois et, dans une certaine mesure, de la poésie profane et du folklore.

7 Le deuxième chapitre du livre présente les événements de la vie de Mikael Agricola et les éléments centraux de son œuvre. L'auteure y analyse également pourquoi et comment Mikael Agricola est devenu un réformateur protestant et le fondateur de la langue finnoise écrite imprimée. Kaisa Häkkinen souligne que nous n'avons que peu d'informations sur Mikael Agricola en tant qu'individu. L'ouvrage présente cependant de manière hypothétique, à partir des informations existantes, le contexte historique et les endroits et institutions où Mikael Agricola a grandi et où il a plus tard exercé son influence. Mikael Agricola n'a pas lui-même décrit ses études et son séjour à Wittenberg, mais quelques informations peuvent être recueillies dans les lettres qu'il a envoyées en 1537 et 1538 à Gustave Vasa. En revanche, nous en savons davantage sur ses activités en Finlande, décrites avec précision dans l'ouvrage. La description de la culture et des conditions d'enseignement dans l'école de la cathédrale de Turku donne un bon aperçu des conditions dans lesquelles Mikael Agricola a rempli son rôle de directeur et montre comment il le considérait. Elle présente également les épreuves qu'il a dû affronter, comme l'incendie de Turku, la mort de ses compagnons de travail et son éviction de la position de directeur. De la même façon, elle propose une bonne description des activités de Mikael Agricola autres que son travail de directeur.

Kaisa Häkkinen mentionne de nombreux contemporains finlandais de Mikael Agricola, qui, comme lui, ont étudié à Wittenberg, et elle présente des individus qui ont probablement influencé Mikael Agricola et son œuvre. Les éléments biographiques du deuxième chapitre de l'ouvrage sont complétés et approfondis par le chapitre 5 , où Kaisa Häkkinen présente de manière plus précise les soutiens et les modèles de Mikael Agricola, de Viktor Bertili de Pernaja à Martin Luther, Philipp Melanchthon ou Érasme de Rotterdam. Ce dernier, bien que Mikael Agricola ne l'ait jamais rencontré personnellement, a été pour lui un modèle important. Kaisa Häkkinen souligne l'importance des relations de Mikael Agricola: sa montée aux positions de décision dans l'Église a été possible non seulement grâce à son talent et à son érudition mais aussi grâce à ses soutiens importants et à ses compagnons de travail.

La Réforme a conduit à un affaiblissement du pouvoir de l'Église dont une grande partie des propriétés ont été transférées à la couronne. L'ouvrage présente ce changement et l'influence du roi Gustave Vasa dans la vie et la carrière de Mikael Agricola dans les chapitres 2 et 5 . La déception de Mikael Agricola à l'égard de la nouvelle séparation du pouvoir religieux et du pouvoir temporel lui a causé des tensions avec le roi, ce qui n'a pas été sans influence sur sa carrière. Le soutien financier n'a pas été constant et son opposition aux commandements du roi a pu être l'une des raisons de son éviction de la fonction de directeur de l'école de la cathédrale. Même en tant qu'évêque, Mikael Agricola n'a pu profiter de la position de pouvoir que les évêques de la Finlande médiévale avaient eue, puisque, avec l'affaiblissement du pouvoir de l'Église, les droits de l'évêque ont également été réduits. Avant que Mikael Agricola ne devienne évêque, le roi avait divisé la Finlande en deux évêchés, ayant chacun leur évêque. En devenant évêque, Mikael Agricola a eu à s'occuper du plus grand des deux évêchés, celui de Turku. L'évêché de Vyborg a été confié à Paulus Juusten. dernier voyage, pour les pourparlers de paix à Moscou. L'ambassade envoyée par le roi 
a réussi dans sa mission et la paix a été conclue, mais pendant le voyage de retour, Mikael Agricola est tombé malade et est mort rapidement. Le chapitre 5 décrit les phases de ce voyage et évalue le rôle de Mikael Agricola comme messager de paix et diplomate. Il n'y a pas d'information certaine sur son rôle dans les pourparlers à Moscou, mais Kaisa Häkkinen estime qu'il ne faut pas exagérer ses mérites dans la sphère de la diplomatie politique. Mikael Agricola n'avait en effet pas d'expérience des pourparlers politiques avant son voyage. Kaisa Häkkinen remarque également qu'il n'était certainement pas très diplomate par nature : il agissait souvent contre les désirs de Gustave Vasa. En outre, dans les préfaces de ses ouvrages, il lui arrivait de répondre à ses opposants et de défendre ses traductions d'une manière tout à fait agressive. D'un autre côté, Kaisa Häkkinen souligne que Mikael Agricola a exercé avec patience sa fonction religieuse de figure de proue de la Réforme et qu'il y a réussi. Les effusions de sang ont été évitées, contrairement à ce qui s'est passé en Allemagne, pays d'origine de la Réforme. Kaisa Häkkinen met également au crédit de Mikael Agricola de ne pas avoir détruit tout l'ancien héritage chrétien datant de l'ère catholique, mais d'avoir uni le nouveau et l'ancien en conservant les dogmes importants et les prières dans la forme qui avait toujours été la leur.

11 Le chapitre 5 présente, outre les relations de Mikael Agricola, sa bibliothèque personnelle qui a été bien conservée. Sur la base de ses livres, on peut affirmer que Mikael Agricola était intéressé par la médecine, l'histoire et la géographie. Kaisa Häkkinen souligne son vaste savoir dans de nombreux domaines. Elle trouve regrettable que, bien qu'il ait dirigé pendant dix ans l'établissement d'enseignement le plus important de Finlande et en ait été le principal enseignant, aucun de ses élèves n'ait laissé de notes sur l'enseignant. Les générations suivantes n'ont ainsi pas de témoignage de la vision qu'avaient de Mikael Agricola ses élèves. Kaisa Häkkinen suppose qu'il a dû dans tous les cas avoir une grande influence également sur les générations futures d'étudiants; son œuvre imprimée faisait de lui une autorité et un modèle dans le domaine de l'utilisation écrite du finnois.

Les chapitres 3 et 4 sont consacrés à une présentation des ouvrages de Mikael Agricola et de leur langue. Dans le chapitre 3, Kaisa Häkkinen présente les neuf ouvrages de Mikael Agricola dans leur ordre de parution ainsi que les principaux textes sources en langue étrangère. Kaisa Häkkinen montre la grande importance des ouvrages de Mikael Agricola en leur temps : plus de 20 ans se sont écoulés avant la publication d'autres livres en finnois. Au début du chapitre, Kaisa Häkkinen souligne également l'importance de la littérature imprimée pour la création d'une langue écrite commune. Il a fallu que des ouvrages soient disponibles en des centaines de copies pour que commence à se former une idée générale de la manière de traiter des sujets à l'écrit. Les textes imprimés étaient également des modèles pour les traducteurs et auteurs postérieurs. Kaisa Häkkinen présente l'histoire de l'impression des ouvrages de Mikael Agricola telle qu'on la connaît et décrit de façon très détaillée le contenu et la structure des ouvrages. Les titres en sont mentionnés à la fois dans leur orthographe originale et dans l'orthographe contemporaine, et les différences linguistiques sont interprétées. Kaisa Häkkinen mentionne également les chercheurs contemporains spécialistes de Mikael Agricola et donne des informations sur la transmission des textes jusqu'à nos jours. Par exemple, il n'est resté aucun exemplaire complet du premier ouvrage de Mikael Agricola, Abckiria, qui a été ultérieurement reconstitué à partir de fragments conservés dans différentes publications. En revanche, la traduction du Nouveau Testament a été conservée dans sa version originale et 59 exemplaires en sont 
conservés dans les bibliothèques publiques de Finlande, sans compter les exemplaires des collections privées.

Bien que le contenu des ouvrages de Mikael Agricola soit principalement religieux, cette liste comprend également des sujets plus profanes. Dans la présentation du deuxième ouvrage de Mikael Agricola, Rucouskiria Bibliasta, Kaisa Häkkinen décrit en l'interprétant la partie calendrier du livre, nommée "première encyclopédie » en finnois parce qu'elle contient des connaissances significatives dans différents domaines. L'auteure présente également la métrique des poèmes de la préface du Rucouskiria. Kaisa Häkkinen ne considère pas Mikael Agricola comme un poète de talent, mais estime que ses poèmes sont « contextuellement colorés et intéressants » et qu'ils dévoilent les rouages de sa pensée lorsqu'il se livrait au travail littéraire. Dans ses poèmes, Mikael Agricola critique par exemple les prêtres paresseux qui ne font pas l'effort d'éduquer le peuple. Il se défend également face à ceux qui se moquent de ses livres et les rabaissent. Le long poème de présentation du dernier ouvrage de Mikael Agricola, Ne Prophetat Haggai SacharJa Maleachi, dévoile ses pensées personnelles sur l'avenir de la lecture en finnois. Mikael Agricola y prédit que bientôt arrivera une époque où l'on voudra lire de plus en plus des livres en finnois mais qu'il sera difficile de s'en procurer. Visiblement déçu par le faible accueil fait à ses œuvres, Mikael Agricola exhorte à lire des livres en finnois tant que c'est encore possible.

Dans le quatrième chapitre de l'ouvrage, le plus long, Kaisa Häkkinen présente la langue des ouvrages de Mikael Agricola sous différents angles : différentes parties du chapitre sont consacrées respectivement à la phonologie, la morphologie, la syntaxe et au lexique. Elle présente également les particularités du finnois aux lecteurs qui ne connaîtraient pas la langue, comme l'importance de la longueur des sons et la correspondance de l'orthographe et de l'articulation dans le finnois contemporain. Kaisa Häkkinen souligne que le développement de la langue vers sa forme contemporaine est un processus de longue durée et qu'on ne peut pas lire les textes de Mikael Agricola comme du finnois contemporain. À ses débuts, Mikael Agricola ne respectait pas la correspondance entre les lettres et les sons propres au finnois contemporain. L'instabilité de l'orthographe de Mikael Agricola est également un signe de ce que, à son époque, la variation était considérée comme un élément naturel non seulement de la langue parlée mais aussi de la langue écrite. Kaisa Häkkinen explique également l'arrière-plan des particularités syntaxiques de la langue de Mikael Agricola. À son époque, la fidélité au texte d'origine était un principe important. Contrairement à aujourd'hui, ce principe concernait également la structure du texte, que l'on modifiait aussi peu que possible. La langue de Mikael Agricola, avec son ordre des mots et sa structure de phrases étranges, diffère donc beaucoup du finnois contemporain, qui ne connaît plus ces étrangetés.

Les caractéristiques de l'orthographe, de la morphologie et de la syntaxe de Mikael Agricola sont étudiées dans une comparaison directe avec le finnois contemporain, ce qui aide les lecteurs, même les lecteurs finnophones, à se figurer le développement du finnois, ainsi que la structure de la langue de Mikael Agricola, comme celle du finnois contemporain. Kaisa Häkkinen présente d'abord les caractéristiques linguistiques en finnois contemporain, avant de les comparer aux caractéristiques correspondantes chez Mikael Agricola. Le lecteur non spécialiste du finnois est pris en compte de nombreuses façons. Les exemples sont traduits en anglais et la compréhension de la structure des mots est facilitée par la séparation des 
morphèmes qui composent les formes déclinées. Les abréviations et symboles utilisés sont listés au début du livre. À la fin de l'ouvrage se trouvent des tableaux de déclinaison et conjugaison du finnois contemporain. Dans la présentation de la syntaxe, les phrases ou parties de phrase utilisées comme exemples sont indiquées par un alinéa, avec leur traduction anglaise, pour être séparées du reste du texte. En outre, le point qui doit attirer l'attention est en gras. Les traductions des phrases se fondent sur une comparaison soigneuse : les exemples provenant de la Bible ont été traduits par des phrases tirées de traductions anglaises de la Bible qui correspondaient le mieux au texte de Mikael Agricola.

En présentant le lexique, Kaisa Häkkinen rend compte qu'on ne peut pas évaluer l'importance du lexique de Mikael Agricola uniquement en se fondant sur le nombre de mots, parce qu'une partie considérable du lexique qui s'est fixé dans la langue écrite par le truchement des ouvrages de Mikael Agricola est un lexique atemporel, dont on ne peut se passer même en finnois contemporain. Des cent mots les plus utilisés dans la langue contemporaine, seuls trois ne se trouvent pas chez Mikael Agricola : eräs (un, un certain), eri (divers) et esittää (présenter). Une estimation du nombre de mots dans les ouvrages de Mikael Agricola les porte à 8500 , auxquels s'ajoutent encore 500 noms propres. Kaisa Häkkinen écrit que ce nombre peut paraître petit en comparaison des 207000 mots du dictionnaire de finnois contemporain, mais en prenant en compte la limitation des sujets de ses textes, ce nombre est considérable. Kaisa Häkkinen donne également un élément de comparaison internationalement connu: il y a dans les ouvrages de Mikael Agricola environ autant de mots que dans le Kalevala, qui est considéré comme un texte riche et coloré linguistiquement. Kaisa Häkkinen souligne également qu'on ne peut pas prendre les textes de Mikael Agricola comme représentatifs de la langue du $\mathrm{XvI}^{\mathrm{e}}$ siècle, puisqu'ils comprennent de nombreux mots d'origine étrangère ainsi que des expressions créées au moment de la traduction, qui ne se sont jamais fixées ni dans la langue écrite ni dans la langue parlée. En outre, il manque dans les ouvrages de Mikael Agricola le lexique de la vie quotidienne en Finlande, qui n'était pas nécessaire dans des textes religieux. Le lexique de Mikael Agricola donne toutefois au lecteur contemporain des connaissances précieuses sur la réalité de la vie au $x^{2} I^{e}$ siècle: par exemple, le lexique du calendrier du Rucouskiria montre que la lecture et l'écriture d'horoscopes étaient des activités importantes même du temps de Mikael Agricola.

Dans le sixième et dernier chapitre de son ouvrage, Kaisa Häkkinen évalue, du point de vue contemporain, l'héritage laissé par Mikael Agricola aux générations suivantes. Elle commence par rappeler qu'en son temps, Mikael Agricola a rencontré dans son travail des difficultés et des oppositions, et que la réception de ses ouvrages n'avait pas été aussi enthousiaste qu'il l'aurait souhaitée. À son époque, et encore longtemps après lui, jusqu'au XIX ${ }^{e}$ siècle, la position du finnois comme langue de culture complète est restée incertaine. Même en tant qu'individu, Mikael Agricola a été oublié après sa mort, en particulier après la parution de la traduction de la Bible en entier en 1642. Dans la préface de cette traduction, la première traduction du Nouveau Testament était certes mentionnée, mais pas le nom de son auteur.

18 Mikael Agricola n'a été retrouvé qu'à la fin du XviII ${ }^{\mathrm{e}}$ siècle, lorsque s'est amorcée la recherche critique sur l'histoire de la Finlande et que les débuts du finnois écrit ont commencé à intéresser les chercheurs. Le chapitre 6 comprend une présentation historique de la recherche sur Mikael Agricola et sur son œuvre. Les premiers 
chercheurs à s'intéresser à son travail, et qui l'ont aussi rendu célèbre, au XvIII ${ }^{e}$ siècle, sont Carl Fredrik Mennander et son élève Henrik Gabriel Porthan. Le rôle de Mikael Agricola comme fondateur de la langue écrite finnoise était connu et reconnu déjà à l'époque, mais la recherche proprement dite sur lui n'a toutefois commencé qu'à la fin du $\mathrm{XIX}^{\mathrm{e}}$ siècle. Kaisa Häkkinen mentionne Gustav Erik Eurén, premier auteur finlandais qui a fait connaître le nom de Mikael Agricola auprès du grand public. August Ahlqvist, quant à lui, professeur de finnois à l'université d'Helsinki, a été le premier à se pencher en détail, à la fin du XIX ${ }^{e}$ siècle, sur la langue de Mikael Agricola. Arvid Genetz et Emil Nestor Setälä comptent parmi ses successeurs les plus célèbres. À la même époque que les recherches d'Ahlqvist, Vicar J. A. Cederberg a écrit une recherche en histoire religieuse sur l'histoire de la Bible finlandaise, qui traitait également de Mikael Agricola. À la fin du XIx ${ }^{\mathrm{e}}$ siècle et au début du Xx $\mathrm{x}^{\mathrm{e}}$ siècle, le nombre de chercheurs travaillant sur Mikael Agricola a augmenté, et Kaisa Häkkinen nomme et présente rapidement les noms de ceux-ci dans les domaines de la linguistique, de la littérature, de la théologie et de l'archéologie. Elle décrit en outre la grande diversification de la recherche linguistique sur Mikael Agricola à la fin $\mathrm{du} \mathrm{xx}^{\mathrm{e}}$ siècle et au début du xxI ${ }^{e}$ siècle, ainsi que la numérisation de la recherche et des matériaux de recherche au début du xxi siècle. La recherche linguistique la plus récente compare de façon systématique les ouvrages de Mikael Agricola aux manuscrits finnois de son époque. $\mathrm{Au} \mathrm{xxI}^{\mathrm{e}}$ siècle ont été également réalisés de nombreux travaux interdisciplinaires dans lesquels se sont joints aux linguistes, aux théologiens et aux historiens de la religion des archéologues, des chercheurs en histoire culturelle, en étude des religions, en littérature, en médecine et botanique.

Dans le dernier chapitre, Kaisa Häkkinen évalue aussi l'importance de Mikael Agricola pour la littérature spirituelle ultérieure. La production de Mikael Agricola satisfaisait presque tous les besoins de l'Église, mais il n'avait pas publié de livre de versets. Cependant, dans les livres de culte évangélico-luthériens finnois contemporains, se trouvent huit versets qui sont d'une façon ou d'une autre liés aux textes traduits par Mikael Agricola, même si aucun d'entre eux n'est tout à fait dans la forme qu'il avait dans les textes originaux. Les psaumes traduits par Mikael Agricola ont été chantés sous leur forme originale ou comme parties d'autres textes de livres de culte. La pierre de touche du travail du comité de traduction de la Bible de 1642 a été la traduction du Nouveau Testament de Mikael Agricola, bien que son orthographe et des bizarreries phonétiques aient été corrigées. Kaisa Häkkinen souligne que, sans compter les nouvelles traductions, l'influence de Mikael Agricola a été considérable dans toute la littérature spirituelle ultérieure, puisque son lexique religieux fondamental, beaucoup de ses traits essentiels et de ses formes lexicales sont originaires de ses ouvrages. La langue religieuse est conservatrice et garde des traits qui disparaissent du reste de la langue écrite.

De nos jours, tous les Finlandais connaissent le nom de Mikael Agricola et savent qu'il est le fondateur de la langue écrite. À la fin du dernier chapitre, Kaisa Häkkinen éclaire la visibilité de Mikael Agricola et son rôle dans la culture contemporaine. Elle mentionne une fiction écrite sur lui, divers projets de statues, et sa présence jusque dans les pages du journal Aku Ankka (Donald Duck). Lorsque, en 2004, les Finlandais ont voté pour le Finlandais le plus important de tous les temps, Mikael Agricola est arrivé à la huitième place. Kaisa Häkkinen estime que Mikael Agricola est devenu un label finlandais, que l'on utilise pour servir l'éducation, la culture, et même le business. 


\section{INDEX}

Keywords : language history, reformation, written culture, written language

Index chronologique : XVe siècle, XVIe siècle, XVIIe siècle, XVIIIe siècle, XIXe siècle, XXe siècle Thèmes : histoire de la langue

nomsmotscles Finnois

motscleset keele ajalugu, kirjakeel, kirjakultuur, reformatsioon

motsclesru ПИСЬМЕННОСТЬ, ИСТОРИЯ ЯЗЫКА, ПИСЬМЕННАЯ КУЛЬТУРА, РЕФОРМАЦИЯ

Mots-clés : langue écrite, histoire de la langue, réforme protestante, culture écrite

disciplines bas-allemand, finnois, latin, same, suédois

Index géographique : Allemagne, Danemark, Finlande, Häme, Helsinki, Lübeck, Pernaja, Suède, Turku, Vyborg, Wittenberg 